PROCEEDINGS OF THE

AMERICAN MATHEMATICAL SOCIETY

Volume 135, Number 10, October 2007, Pages 3375-3381

S 0002-9939(07)08852-1

Article electronically published on June 20, 2007

\title{
REMARK ABOUT SCALAR CURVATURE AND RIEMANNIAN SUBMERSIONS
}

\author{
JOHN LOTT
}

(Communicated by Jon G. Wolfson)

\begin{abstract}
We consider modified scalar curvature functions for Riemannian manifolds equipped with smooth measures. Given a Riemannian submersion whose fiber transport is measure-preserving up to constants, we show that the modified scalar curvature of the base is bounded below in terms of the scalar curvatures of the total space and fibers. We give an application concerning scalar curvatures of smooth limit spaces arising in bounded curvature collapses.
\end{abstract}

\section{INTRODUCTION}

An important result in the study of manifolds of nonnegative sectional curvature is O'Neill's theorem, which says that sectional curvature is nondecreasing under a Riemannian submersion [1, Chapter 9]. In a previous paper we showed that there is a Ricci analog of O'Neill's theorem, provided that one uses a modified Ricci tensor and assumes that the fiber transport of the Riemannian submersion preserves measures up to multiplicative constants [6, Theorem 2]. We used this to show that, in the case of a bounded curvature collapse with a smooth limit space, a lower Ricci bound on the collapsing manifolds implies a lower bound on the modified Ricci curvature of the limit space [6, Theorem 3].

These results can be viewed as precursors to a synthetic treatment of Ricci curvature for metric-measure spaces [7, 12. In particular, [6, Theorem 2] is given a synthetic proof in [7, Corollary 7.52], and [6, Theorem 3] follows from [7, Corollary 7.45]. (To be precise, with the notation of [7], if $N<\infty$, then one must restrict to the case of nonnegative Ricci curvature.)

In the present note we consider scalar curvature analogs of [6, Theorems 2 and 3]. A modified scalar curvature function for a smooth Riemannian manifold, equipped with a smooth measure, was defined by Perelman [8, Section 1.3]. Let $(M, g)$ be a Riemannian manifold. Let $\mathrm{dvol}_{M}$ denote the Riemannian density on $M$. Let $\phi$ be a smooth positive function on $M$. Then $\left(M, \phi \operatorname{dvol}_{M}\right)$ is a smooth metric-measure space. Perelman's modified scalar curvature is

$$
R_{\infty}=R-2 \frac{\nabla^{2} \phi}{\phi}+\frac{|\nabla \phi|^{2}}{\phi^{2}} .
$$

Received by the editors May 10, 2005 and, in revised form, July 3, 2006.

2000 Mathematics Subject Classification. Primary 53C21; Secondary 58G25.

Research supported by NSF grant DMS-0306242 and the Miller Institute.

(C)2007 American Mathematical Society 
It is designed so that a weighted Lichnerowicz identity holds. In particular, if $M$ is a closed spin manifold and $R_{\infty}>0$, then $\widehat{A}(M)=0$. Perelman's entropy functional is the corresponding total scalar curvature, i.e. $\mathcal{F}=\int_{M} R_{\infty} \phi \operatorname{dvol}_{M}$. Note that $R_{\infty}$ is not the trace of the Bakry-Émery tensor $\operatorname{Ric}_{\infty}=\operatorname{Ric}-\frac{\operatorname{Hess} \phi}{\phi}+\frac{d \phi}{\phi} \otimes \frac{d \phi}{\phi}$.

More generally, for $q \in(0, \infty)$, we define

$$
R_{q}=R-2 \frac{\nabla^{2} \phi}{\phi}+\left(1-\frac{1}{q}\right) \frac{|\nabla \phi|^{2}}{\phi^{2}} .
$$

We now state a scalar curvature analog of the Ricci O'Neill theorem of 6 , Theorem 2]. Suppose that a Riemannian submersion $p: M \rightarrow B$ has compact fiber $F$. Put $F_{b}=p^{-1}(b)$. Given a smooth curve $\gamma:[0,1] \rightarrow B$ and a point $m \in F_{\gamma(0)}$, let $\rho(m)$ be the endpoint $\bar{\gamma}(1)$ of the horizontal lift $\bar{\gamma}$ of $\gamma$ that starts at $m$. Then $\rho$ is the fiber transport diffeomorphism from $F_{\gamma(0)}$ to $F_{\gamma(1)}$.

Given the positive function $\phi^{M}$ on $M$, define $\phi^{B}$, a smooth positive function on $B$, by

$$
p_{*}\left(\phi^{M} \operatorname{dvol}_{M}\right)=\phi^{B} \operatorname{dvol}_{B} .
$$

Let $\phi^{F}$ denote the restriction of $\phi^{M}$ to a fiber $F$. Let $R_{\infty}^{M}, R_{\infty}^{B}, R_{q}^{B}$ and $R_{\infty}^{F}$ denote the modified scalar curvature functions. Let $\mathrm{dvol}_{F}$ denote the fiberwise Riemannian density. Let $\int_{F}$ denote fiberwise integration.

Theorem 1.1. Suppose that fiber transport preserves the fiberwise measure $\phi^{F} \mathrm{dvol}_{F}$ up to multiplicative constants, i.e. for any smooth curve $\gamma:[0,1] \rightarrow B$, there is a

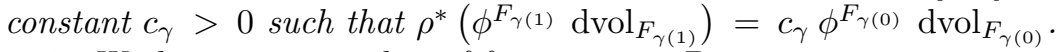

1. We have an inequality of functions on $B$ :

$$
R_{\infty}^{B} \geq \frac{\int_{F}\left(R_{\infty}^{M}-R_{\infty}^{F}\right) \phi^{M} \mathrm{dvol}_{F}}{\int_{F} \phi^{M} \operatorname{dvol}_{F}} .
$$

2. Suppose in addition that $\phi^{M}=1$. Put $q=\operatorname{dim}(F)$. Then

$$
R_{q}^{B} \geq \frac{\int_{F}\left(R^{M}-R^{F}\right) \mathrm{dvol}_{F}}{\operatorname{vol}(F)}
$$

We now give an application of Theorem 1.1 to collapsing of Riemannian manifolds. A general phenomenon in collapsing is that when the limit space is smooth, a lower curvature bound on the collapsing manifolds tends to give a lower curvature bound on the limit space. This is clearly not true for scalar curvature, as can be seen by taking a product $B \times S^{q}$, with $q>1$, and shrinking the $S^{q}$-factor. One can get around this particular problem by assuming an upper bound on the scalar curvatures of the collapsing manifolds. The next result is a scalar curvature analog of [6. Theorem 3].

Theorem 1.2. Let $\left\{\left(M_{i}, g_{i}\right)\right\}_{i=1}^{\infty}$ be a sequence of $N$-dimensional connected closed Riemannian manifolds with sectional curvatures bounded above in absolute value by $\Lambda$ and diameters bounded above by $D$, for some $D, \Lambda \in \mathbb{R}^{+}$. Let $(X, \mu)$ be a limit point for $\left\{\left(M_{i}, g_{i}\right)\right\}_{i=1}^{\infty}$ in the measured Gromov-Hausdorff topology. Suppose that for some $r \in \mathbb{R}$ and all $i \in \mathbb{Z}^{+}, R\left(M_{i}, g_{i}\right) \geq r$. Suppose that $X$ is an $n$-dimensional closed manifold. Put $q=N-n$.

a. If $q=0$, then $X$ has $R \geq r$.

b. If $q>0$, then $X$ has $R_{q} \geq r$. Consequently, $\frac{\int_{X} R^{X} \mathrm{dvol}_{X}}{\operatorname{vol}(X)} \geq r$. 
In the statement of Theorem 1.2, the pair $\left(g_{X}, \phi^{X}\right)$ is $W^{2, p}$-regular for all $p \in$ $[1, \infty)$. Hence $R$ and $R_{q}$ are well defined in $\bigcap_{p \in[1, \infty)} L^{p}(X)$.

The last inequality in Theorem 1.2 can be compared with the lower bound in [7. Corollary 7.49] on the mean scalar curvature of a smooth limit space when it arises from a sequence of collapsing manifolds with Ricci curvature bounded below by $K \in \mathbb{R}$. One could ask whether there is an analog of Theorem 1.2 if one assumes a two-sided bound on the Ricci curvature of the manifolds $M_{i}$, as opposed to the sectional curvature.

I thank the UC-Berkeley Mathematics Department for its hospitality while this note was written.

\section{Proof of Theorem 1.1}

We use the notation of [6, Section 3], which in turn uses the notation of [1, Chapter 9]. If $X$ is a vector field on $B$, let $\bar{X}$ be its horizontal lift to $M$. Let $N$ be the mean curvature vector field to the fibers $F$. Let $A$ be the curvature of the horizontal distribution. Let $T$ be the second fundamental form tensor of the fibers $F$. Let $\nabla^{M}$ be the covariant derivative operator on $M$ and let $\nabla^{B}$ be the covariant derivative operator on $B$. Define $\check{\delta} N \in C^{\infty}(M)$ by saying that if $\left\{\bar{e}_{\alpha}\right\}$ is an orthonormal basis of $T_{h o r} M$ at a point $m$, then $(\check{\delta} N)(m)=-\sum_{\alpha}\left\langle\nabla_{\bar{e}_{\alpha}}^{M} N, \bar{e}_{\alpha}\right\rangle$. From [1, Corollary 9.37], there is an identity of functions on $M$ :

$$
R^{M}=R^{B}+R^{F}-|A|^{2}-|T|^{2}-|N|^{2}-2 \check{\delta} N .
$$

Given $f \in C^{\infty}(M)$, we write $\nabla_{M}^{2} f$ for the Laplacian of $f$. Define $\nabla_{\text {hor }}^{2} f \in$ $C^{\infty}(M)$ by saying that $\left(\nabla_{h o r}^{2} f\right)(m)=\sum_{\alpha}($ Hess $f)\left(\bar{e}_{\alpha}, \bar{e}_{\alpha}\right)$. Define $\nabla_{F}^{2} f \in C^{\infty}(M)$ by saying that if $m$ is in a fiber $F$, then $\left(\nabla_{F}^{2} f\right)(m)$ is the Laplacian of the function $\left.f\right|_{F}$ on $F$, evaluated at $m$. One can check that

$$
\nabla_{M}^{2} f=\nabla_{h o r}^{2} f+\nabla_{F}^{2} f-\left(\nabla_{h o r} f, N\right) .
$$

Given $b \in B$, let $\left\{\theta_{t}\right\}_{t \in(-\epsilon, \epsilon)}$ be the flow of $X$ as defined in a neighborhood of $b$ and for $t$ in some interval $(-\epsilon, \epsilon)$. Let $\left\{\bar{\theta}_{t}\right\}_{t \in(-\epsilon, \epsilon)}$ be the flow of $\bar{X}$ that covers $\theta_{t}$. It sends fibers to fibers diffeomorphically. Hence it makes sense to define $\mathcal{L}_{\bar{X}} \mathrm{dvol}_{F}$ by

$$
\left.\left(\mathcal{L}_{\bar{X}} \operatorname{dvol}_{F}\right)\right|_{F_{b}}=\left.\left.\frac{d}{d t}\right|_{t=0}\left(\bar{\theta}_{t}^{*} \operatorname{dvol}_{F}\right)\right|_{F_{b}} .
$$

With our conventions,

$$
\mathcal{L}_{\bar{X}} \mathrm{dvol}_{F}=-(\bar{X}, N) \mathrm{dvol}_{F} .
$$

We have

$$
\phi^{B}=\int_{F} \phi^{M} \mathrm{dvol}_{F}
$$

Then

$$
\begin{aligned}
X \phi^{B} & =\mathcal{L}_{X} \phi^{B}=\mathcal{L}_{X} \int_{F} \phi^{M} \operatorname{dvol}_{F}=\int_{F} \mathcal{L}_{\bar{X}}\left(\phi^{M} \mathrm{dvol}_{F}\right) \\
& =\int_{F}\left(\bar{X} \phi^{M}-(\bar{X}, N) \phi^{M}\right) \mathrm{dvol}_{F}
\end{aligned}
$$


From [6, (3.7)],

$$
\begin{gathered}
\operatorname{Hess}\left(\phi^{B}\right)(X, X)=\int_{F}\left[\frac{\operatorname{Hess}\left(\phi^{M}\right)(\bar{X}, \bar{X})}{\phi^{M}}-\left(\frac{\bar{X} \phi^{M}}{\phi^{M}}\right)^{2}-\left(\bar{X}, \nabla \frac{M}{X} N\right)\right. \\
\left.+\left(\frac{\bar{X} \phi^{M}}{\phi^{M}}-(\bar{X}, N)\right)^{2}\right] \phi^{M} \mathrm{dvol}_{F} .
\end{gathered}
$$

Then

$$
\nabla_{B}^{2} \phi^{B}=\int_{F}\left[\frac{\nabla_{h o r}^{2} \phi^{M}}{\phi^{M}}-\left|\frac{\nabla_{h o r} \phi^{M}}{\phi^{M}}\right|^{2}+\check{\delta} N+\left|\frac{\nabla_{h o r} \phi^{M}}{\phi^{M}}-N\right|^{2}\right] \phi^{M} \operatorname{dvol}_{F} .
$$

Given $b \in B$, put $F_{b}=p^{-1}(b)$. From (1.1), (2.1) and (2.8),

$$
\begin{aligned}
\phi^{B}(b) R_{\infty}^{B}(b)= & \phi^{B}(b) R^{B}(b)-2 \nabla_{B}^{2} \phi^{B}+\frac{\left|\nabla_{B} \phi^{B}\right|^{2}}{\phi^{B}} \\
= & \int_{F_{b}} R^{B} \phi^{M} \operatorname{dvol}_{F}-2 \nabla_{B}^{2} \phi^{B}+\frac{\left|\nabla_{B} \phi^{B}\right|^{2}}{\phi^{B}} \\
= & \int_{F_{b}}\left[\left(R^{M}-R^{F}+|A|^{2}+|T|^{2}+|N|^{2}\right) \phi^{M}\right. \\
& \left.-2 \nabla_{h o r}^{2} \phi^{M}+2 \frac{\left|\nabla_{h o r} \phi^{M}\right|^{2}}{\phi^{M}}-2\left|\frac{\nabla_{h o r} \phi^{M}}{\phi^{M}}-N\right|^{2} \phi^{M}\right] \operatorname{dvol}_{F} \\
& +\frac{\left|\nabla_{B} \phi^{B}\right|^{2}}{\phi^{B}} .
\end{aligned}
$$

We have

$$
\mathcal{L}_{\bar{X}}\left(\phi^{M} \operatorname{dvol}_{F}\right)=\left(\frac{\bar{X} \phi^{M}}{\phi^{M}}-(\bar{X}, N)\right) \phi^{M} \operatorname{dvol}_{F}
$$

By assumption, $\frac{\bar{X} \phi^{M}}{\phi^{M}}-(\bar{X}, N)$ is constant on a fiber $F$. Let $\left\{e_{\alpha}\right\}$ be an orthonormal basis of $T_{b} B$ and let $\left\{\bar{e}_{\alpha}\right\}$ be the horizontal lift of $\left\{e_{\alpha}\right\}$ along $F_{b}$. Then

$$
\begin{aligned}
\frac{\left(e_{\alpha} \phi^{B}\right)^{2}}{\phi^{B}} & =\left(\phi^{B}\right)^{-1}\left(\int_{F_{b}}\left(\frac{\bar{e}_{\alpha} \phi^{M}}{\phi^{M}}-\left(\bar{e}_{\alpha}, N\right)\right) \phi^{M} \mathrm{dvol}_{F}\right)^{2} \\
& =\int_{F_{b}}\left(\frac{\bar{e}_{\alpha} \phi^{M}}{\phi^{M}}-\left(\bar{e}_{\alpha}, N\right)\right)^{2} \phi^{M} \operatorname{dvol}_{F},
\end{aligned}
$$

and so

$$
\frac{\left|\nabla_{B} \phi^{B}\right|^{2}}{\phi^{B}}=\int_{F_{b}}\left|\frac{\nabla_{h o r}^{M} \phi^{M}}{\phi^{M}}-N\right|^{2} \phi^{M} \operatorname{dvol}_{F} .
$$


From (2.9) and (2.12),

$$
\begin{aligned}
\phi^{B}(b) R_{\infty}^{B}(b)=\int_{F_{b}} & {\left[\left(R^{M}-R^{F}+|A|^{2}+|T|^{2}+|N|^{2}\right) \phi^{M}\right.} \\
& \left.-2 \nabla_{h o r}^{2} \phi^{M}+2 \frac{\left|\nabla_{h o r} \phi^{M}\right|^{2}}{\phi^{M}}-\left|\frac{\nabla_{h o r} \phi^{M}}{\phi^{M}}-N\right|^{2} \phi^{M}\right] \mathrm{dvol}_{F} \\
=\int_{F_{b}} & {\left[\left(R^{M}-R^{F}+|A|^{2}+|T|^{2}\right) \phi^{M}-2 \nabla_{h o r}^{2} \phi^{M}\right.} \\
+ & \left.\frac{\left|\nabla_{h o r} \phi^{M}\right|^{2}}{\phi^{M}}+2\left(\nabla_{h o r} \phi^{M}, N\right)\right] \mathrm{dvol}_{F} .
\end{aligned}
$$

As

$$
\left|\nabla_{M} \phi^{M}\right|^{2}=\left|\nabla_{h o r} \phi^{M}\right|^{2}+\left|\nabla_{F} \phi^{M}\right|^{2}
$$

we can combine (2.13) with (2.2) and (2.14) to obtain

$$
\begin{aligned}
\phi^{B}(b) R_{\infty}^{B}(b) & =\int_{F_{b}}\left(R_{\infty}^{M}-R_{\infty}^{F}+|A|^{2}+|T|^{2}\right) \phi^{M} \operatorname{dvol}_{F} \\
& \geq \int_{F_{b}}\left(R_{\infty}^{M}-R_{\infty}^{F}\right) \phi^{M} \operatorname{dvol}_{F} .
\end{aligned}
$$

This proves Theorem 1.1,1.

Now suppose that $\phi^{M}=1$. From (2.12), (2.15) and the definition of $R_{q}$ from (1.2),

$$
\begin{aligned}
\phi^{B}(b) R_{q}^{B}(b) & =\phi^{B}(b) R_{\infty}^{B}(b)-\frac{1}{q} \frac{\left|\nabla_{B} \phi^{B}\right|^{2}}{\phi^{B}} \\
& =\int_{F_{b}}\left(R^{M}-R^{F}+|A|^{2}+|T|^{2}-\frac{1}{q}|N|^{2}\right) \mathrm{dvol}_{F} .
\end{aligned}
$$

As $\left(\bar{e}_{\alpha}, N\right)=-\operatorname{Tr}\left(T \bar{e}_{\alpha}\right)$, we know that $\left(T \bar{e}_{\alpha}, T \bar{e}_{\alpha}\right)-\frac{1}{q}\left(\bar{e}_{\alpha}, N\right)^{2} \geq 0$. Summing over $\alpha$ gives $|T|^{2}-\frac{1}{q}|N|^{2} \geq 0$. This proves Theorem 1.1.2.

\section{Proof of Theorem 1.2}

Let $\left\{M_{i}, g_{i}\right\}_{i=1}^{\infty}$ be a sequence as in the statement of Theorem 1.2. We may assume that $\lim _{i \rightarrow \infty}\left(M_{i}, g_{i}, \operatorname{dvol}_{i}\right)=(X, \mu)$ in the measured Gromov-Hausdorff topology. If $q=0$, then $X$ is a smooth manifold with a $W^{2, p}$-regular metric $g_{X}$; after taking a subsequence and applying diffeomorphisms, we may assume that $\left(M_{i}, g_{i}\right)$ converges to $\left(X, g_{X}\right)$ in the $W^{2, p}$-topology (see, for example, [5, 9, 10]). In particular, $\lim _{i \rightarrow \infty} R\left(g_{i}\right)=R\left(g_{X}\right)$ in $L^{p}(X)$. Thus $R^{X} \geq r$.

Suppose that $q>0$. By saying that $X$ is a manifold, we mean that in the construction of $X$ as a quotient space $\widehat{X} / O(N)$ [4, the action of $O(N)$ on the manifold $\widehat{X}$ has a single orbit type. Then $X$ has the structure of a smooth manifold with a $W^{2, p}$-regular pair $\left(g_{X}, \phi^{X}\right)$. 
For any $\epsilon>0$, we can apply smoothing results of Abresch and others 2, Theorem 1.12] to obtain new metrics $g_{i}(\epsilon)$ with

$$
\begin{aligned}
e^{-\epsilon} g_{i} \leq g_{i}(\epsilon) & \leq e^{\epsilon} g_{i}, \\
\left|\nabla_{g_{i}}-\nabla_{g_{i}(\epsilon)}\right| & \leq \epsilon, \\
\left|\nabla_{g_{i}(\epsilon)}^{k} \operatorname{Riem}\left(M_{i}, g_{i}(\epsilon)\right)\right| & \leq C_{k}(N, \epsilon, \Lambda),
\end{aligned}
$$

where the constants are uniform. We can also assume that $R\left(M_{i}, g_{i}(\epsilon)\right) \geq r-\epsilon$; compare [3, Remark 2, p. 51] and [11, Theorem 2.1]. For small $\epsilon$, let $B(\epsilon)$ be a Gromov-Hausdorff limit of a subsequence of $\left\{\left(M_{i}, g_{i}(\epsilon)\right)\right\}_{i=1}^{\infty}$. We relabel the subsequence as $\left\{\left(M_{i}, g_{i}(\epsilon)\right)\right\}_{i=1}^{\infty}$. From [2, Proposition 4.9], for large $i$, there is a small $C^{2}$-perturbation $g_{i}^{\prime}(\epsilon)$ of $g_{i}(\epsilon)$ which is invariant with respect to a Nilstructure. In particular, we may assume that $R\left(M_{i}, g_{i}^{\prime}(\epsilon)\right) \geq r-2 \epsilon$. Now $\left(M_{i}, g_{i}^{\prime}(\epsilon)\right)$ is the total space of a Riemannian submersion $M_{i} \rightarrow B(\epsilon)$ with infranil fibers and affine holonomy. Let $\left(g_{i}^{B(\epsilon)}, \phi_{i}^{B(\epsilon)}\right)$ denote the induced metric and measure on $B(\epsilon)$. As the fiber transport of the Riemannian submersion preserves the affine-parallel volume forms of the fibers up to multiplicative constants, and the infranil fibers have nonpositive scalar curvature, Theorem 1.12 implies that $R_{q}\left(B(\epsilon), g_{i}^{B(\epsilon)}, \phi_{i}^{B(\epsilon)}\right) \geq$ $r-2 \epsilon$. Varying $i$ and $\epsilon$, we can extract a subsequence of $\left\{\left(B(\epsilon), g_{i}^{B(\epsilon)}, \phi_{i}^{B(\epsilon)}\right)\right\}$ with $i \rightarrow \infty$ and $\epsilon \rightarrow 0$ that converges in the $W^{2, p}$-topology to $\left(X, g_{X}, \phi^{X}\right)$. Thus $R_{q}^{X} \geq r$.

Finally,

$$
R_{q}^{X}=R^{X}-2 \nabla^{2} \ln \left(\phi^{X}\right)-\left(1+\frac{1}{q}\right)\left|\nabla \ln \left(\phi^{X}\right)\right|^{2} .
$$

Then

$$
r \leq \frac{\int_{X} R_{q}^{X} \mathrm{dvol}_{X}}{\operatorname{vol}(X)} \leq \frac{\int_{X} R^{X} \mathrm{dvol}_{X}}{\operatorname{vol}(X)}
$$

\section{REFERENCES}

1. A. Besse, Einstein manifolds, Ergebnisse der Mathematik und ihrer Grenzgebiete (3), vol. 10, Springer-Verlag, Berlin, 1987. MR0867684 (88f:53087)

2. J. Cheeger, K. Fukaya and M. Gromov, Nilpotent structures and invariant metrics on collapsed manifolds, J. Amer. Math. Soc. 5 (1992), 327-372. MR1126118 (93a:53036)

3. X. Dai, G. Wei, and R. Ye, Smoothing Riemannian metrics with Ricci curvature bounds, Manuscripta Math. 90 (1996), 49-61. MR:1387754 (97b:53039)

4. K. Fukaya, A boundary of the set of the Riemannian manifolds with bounded curvatures and diameters, J. Diff. Geom. 28 (1988), 1-21. MR0950552 (89h:53090)

5. A. Kasue, A convergence theorem for Riemannian manifolds and some applications, Nagoya Math. J. 114 (1989), 21-51. MR1001487 (90g:53053)

6. J. Lott, Some geometric properties of the Bakry-Émery-Ricci tensor, Comm. Math. Helv. 78 (2003), 865-883. MR2016700 (2004i:53044)

7. J. Lott and C. Villani, Ricci curvature for metric-measure spaces via optimal transport, to appear, Ann. of Math.

8. G. Perelman, The entropy formula for the Ricci flow and its geometric applications, preprint, http://www.arxiv.org/abs/math.DG/0211159 (2002).

9. S. Peters, Convergence of Riemannian manifolds, Compositio Math. 62 (1987), 3-16. MR 0892147 (88i:53076)

10. P. Petersen, Convergence theorems in Riemannian geometry, Comparison Geometry, MSRI Publ. 30, Cambridge Univ. Press, Cambridge, 1997, pp. 167-202. MR.1452874 (98k:53049) 
11. X. Rong, On the fundamental groups of manifolds of positive sectional curvature, Ann. of Math. 143 (1996), 397-411. MR1381991 (97a:53067)

12. K.-T. Sturm, On the geometry of metric measure spaces. I and II, Acta Math. 196 (2006), 65-131 and 133-177. MR2237206 MR2237207

Department of Mathematics, University of Michigan, Ann Arbor, Michigan 481091043

E-mail address: lott@umich.edu 